\title{
Mathematik in Norwegen III: Die „dänische Zeit" vor Abels Erscheinen
}

\author{
Rolf Tomas Nossum und Reinhard Siegmund-Schultze
}

\begin{abstract}
Die springen und rennen in den Wissenschaften herum, lernen einen Haufen Worte und Fakten, reisen ins Ausland mit schlechten Fundamenten, disputieren und predigen und lassen sich dafür von ihren Kameraden bewundern, messen sich immer an ihren eigenen Landsleuten, die ihrer Meinung nach das Höchste erreicht haben ... Es scheint gleichsam, als wenn die Natur sie auf etwas Niederes beschränkt hat, um so den Bauern im eigenen Lande zu imponieren.
\end{abstract}

Hans Gram ${ }^{1}$

\begin{abstract}
$A k$, men så kom fremmedåget, og forplumred urskogs-sproget. Firehundreårig natten ruged over abekatten; og man ved, SÅ lange netter landsens folk $i$ stampe scetter.
\end{abstract}

Ach, das fremde Joch kam und verdarb die Urwald-Sprache.

Die vierhundertjährige Nacht ruhte über dem Affen und man weiß, dass SO lange Nächte die Einheimischen hemmen.

Henrik Ibsen, Peer Gynt, 4. Akt, Szene 13, 1867 (Unsere Übersetzung)

Während in Deutschland kein Mathematiker vor Gauß mit Leibniz vergleichbar war, hat es vor Niels Henrik Abels Arbeiten der $1820 e r$ Jahre in den skandinavischen Ländern kaum mathematische Forschungstraditionen gegeben. In Dänemark kam erst 1814 mit dem in Braunschweig geborenen aber im Lande ausgebildeten Carl Ferdinand Degen (1766-1825), der später Abel anregte, ein guter Mathematiker an die Kopenhagener Universität. Der Norweger Caspar Wessel (1745-1818), der 1798 fast unbemerkt und vor Gauß eine der Gaußschen mindestens gleichwertige geometrische Repräsentation von Vektoren und komplexen Zahlen unter dem Titel Om directionens analytiske betegning (Über die analytische Darstellung der Richtung) veröffentlichte, war eine der seltenen Ausnahmen eines forschenden Mathematikers vor 1800 (mehr über Wessel unten). Trotz der Sprachbarriere waren deutsche mathematische Lehrbücher, wie die des Schülers von Leibniz, Christian Wolff (1679-1754), und von Gauß' eigenem, wenn auch von ihm wenig geschätzten Lehrer Abraham Gotthelf Kästner (1719-180o), in Skandinavien einflussreich. Deutsche Mathematiklehrer suchten gelegentlich Anstellung im dänischen Reiche, und einige von ihnen, wie Ambrosius Rhodius (Rhode), auf den wir kurz zurückkommen werden, wurde von dort weiter nach Norwegen vermittelt. Es gibt zahlreiche Verflechtungen zwischen der deutschen und der skandinavischen Mathematikgeschichte, von denen einige auch in diesem Artikel zur Sprache kommen werden.
Für die Zeit vor 1800 wird man allerdings in erster Linie nach pädagogischen, theologischen und philosophischen Traditionen und nach Anwendungsfeldern wie Astronomie und Landmessung suchen müssen, wenn man nach der Mathematik in Norwegen fragt. Dies gilt aber eigentlich auch für Dänemark, und man wird für diese Periode letztlich nicht klar zwischen dänischer und norwegischer Mathematik unterscheiden können. Das Verhältnis Dänemarks zu seiner von ihm seit Jahrhunderten administrierten norwegischen Provinz, die die Größe des dänischen Stammlandes um ein Vielfaches übertraf und sich zweieinhalb tausend Kilometer nach dem Norden hin erstreckte, war natürlich auch bildungshistorisch eng. Trotz der starken allgemeinen Wirkungen von Reformation und Aufklärung aus dem Süden, der vielfachen dynastischen Verflechtungen zwischen den deutschen und dänischen Adelshäusern, trotz der deutschdänischen Sprachkoexistenzen insbesondere in den damals dänischen Herzogtümern Schleswig und Holstein, war das Verhältnis zwischen Dänemark und Norwegen enger, als zu den deutschen Nachbarstaaten im Süden. Doch war das norwegisch-dänische Verhältnis zugleich politisch problematisch und ist von patriotischen Norwegern zuweilen als eine vierhundertjährige, bis 1814 andauernde „Nacht“ dänischer Fremdherrschaft beschrieben worden. Die klingt noch ein halbes Jahrhundert später in der eingangs zitierten selbstironischen Stelle aus Ibsens Peer Gynt an, und nicht so ironisch 1897 in einem Brief von Sophus Lie an Ludvig Sylow (s. unten). In dem anderen einführenden Zitat ist dagegen einiges von der Arroganz der dänischen Beamten gegenüber der nördlichen Bauernnation zu spüren, deren Söhne für ein Studium ins „Ausland“, insbesondere nach Kopenhagen gehen mussten.

Kurze einführende allgemeinhistorische Bemerkungen zum politischen Verhältnis zwischen Dänemark und Norwegen vor 1800

Die „vierhundertjährige Nacht“ kann man gut als rhetorisches Instrument für nationalistische norwegische Stimmungen im 19. Jahrhundert verstehen, die damals eigentlich 
auf die vollständige nationale Unabhängigkeit von der neuen Vormacht der Schweden gerichtet waren, die schließlich 1905 mit der Konstitution Norwegens als eigene Nation erreicht wurde. Es wurde das Bild eines goldenen Zeitalters bis hinein in das 14. Jahrhundert gezeichnet, das dann zunächst von mehreren Jahrhunderten nationalen Verfalls, danach einem nationalen Wiedererwachen im 18. Jahrhundert und schließlich der relativen Unabhängigkeit seit 1814 mit der Gründung des norwegischen Parlaments (Storting) gefolgt worden sei. Wie wir sehen werden, ist dieses Klischee nicht treffsicher, weder was administrative Reformen, noch was insbesondere die Entwicklung des Faches Mathematik betrifft.

Das 14. Jahrhundert war durch vielfache und wechselnde skandinavische Allianzen gekennzeichnet, wobei Norwegens politische Stellung im Vergleich zu den Nachbarländern allmählich geschwächt wurde. Die Pestkatastrophe von 1347 bis 1351 traf Norwegen besonders hart. Die Regierungszeit von König Olav IV. 1380-1387 leitete eine Reihe von dänisch-norwegischen Allianzen unter dänischer Vorherrschaft ein, die 434 Jahre dauerten, was wohl die Grundlage für Ibsens Ausdruck „vierhundertjährige Nacht“ geworden ist. Die alte norwegische Sprache Norrøn (die Ibsen die „Urwaldsprache“ nennt) wurde zunehmend vor allem mittels Beamtenschaft, Kirche und Schulwesen durch Dänisch ersetzt. ${ }^{2}$

Unter den wechselnden Personalunionen mit den schwedischen und dänischen Königshäusern seit dem Tode des norwegischen Königs Håkon V. im Jahre 1319 (Nossum/Siegmund-Schultze 2011) und bis zur Reformation im 16. Jahrhundert war der norwegische Reichsrat das höchste politische Organ für norwegische adelige und kirchliche Interessen. Die Macht des Reichsrates wurde unter dänischer Vorherrschaft zunehmend beschnitten, insbesondere in den Regierungszeiten von Christian II. (1513-23), Fredrik I. (1523-33) und Christian III. (1534-1559). Christian III. war auf dem Reichstag in Worms 1521 anwesend und wurde ein eifriger Anhänger von Luther. Er hat dann bereits als Lehnsherr von Tönning und Haderslev seit 1525 Luthersche Reformen durchgeführt, bevor er 1534 zum dänischen König gewählt wurde. Die Wahl war umstritten, gerade wegen Christians lutherischer Haltung, und erst seit 1536/37 war er unbestrittener König von Dänemark und Norwegen. Die Macht des norwegischen Reichsrates endete 1536, als Norwegen Provinz Dänemarks auf gleicher Ebene mit Fyn, Skjælland und Skåne wurde. Seitdem kann Norwegen nicht mehr als selbständiger Staat betrachtet werden. Der norwegische Adel und die Geistlichkeit wurden vollständig marginalisiert, im heutigen Norwegen gibt es kaum noch eine Spur von altem norwegischen Adel. Der dänische Reichsrat blieb bis 166 o mächtig, als Fredrik III. die Alleinherrschaft (eneveldet) einführte.

\section{Mathematische Schulbildung in Dänemark-Norwegen vor Abel}

Für unsere Übersicht über die Mathematik in Norwegen stützen wir uns im Folgenden meist auf ältere dänische und norwegische Literatur, die allerdings bisher nicht in Fremdsprachen (Englisch oder Deutsch) übersetzt und kaum durch neuere Darstellungen ergänzt worden ist. Wir erwähnen besonders die Dissertation von Christensen (1895), Nielsen (1912) und das Buch des historisch interessierten bedeutenden norwegischen Zahlentheoretikers Viggo Brun (1885-1978) aus dem Jahre 1962.

Organisierte Schulbildung in Norwegen geht auf das Mittelalter zurück, allerdings beschränkt auf sogenannte Kathedralschulen zur Ausbildung von Priestern in den drei größten Städten Trondheim, Oslo und Bergen. Nach der Reformation galt dänisches Kirchenrecht (1539), die Kathedralschulen wurden umgebildet zu Lateinschulen (obwohl sie oft noch den alten Namen trugen), von denen es bald mehrere zusätzliche gab (Stavanger, Christianssand). Auch die breitere Bevölkerung sollte in Christenlehre erzogen werden, was aber praktisch zunächst zu bescheidenen Resultaten führte. Das erste von einem Norweger in der Muttersprache geschriebene Rechenbuch ist wahrscheinlich das von Tyge Hansøn aus Trondheim aus dem Jahre 1645. Im Vorwort nennt Hansøn es „ein kleines norwegisches Rechenbuch“, aber es wurde unter dem Namen Arithmetica Danica in Kopenhagen gedruckt.

Erst mit wachsender Staatsmacht, mit vom dänischen König bestimmten Beamten, die auch nach Norwegen geschickt wurden und verstärkt durch den religiösen Pietismus im 18. Jahrhundert, begann sich die Administration in Kopenhagen ernsthaft für Erziehungsprobleme zu interessieren. Es gab neue Statuten für die einzige Universität (Kopenhagen 1732) und für die Lateinschulen (1739), sowie Vorschriften für Konfirmation (1736) und damit verbunden allgemeine fünfjährige Schulpflicht (1739). Die Volksschule (allmueskole) gab Unterricht im Christentum, Lesen, Schreiben und Rechnen, die beiden letzten Fächer allerdings auf freiwilliger Basis. An den Lateinschulen und der Universität in Kopenhagen, wo auch Norweger studierten, wurden die Beamten (embetsmenn) und Staats- und Kirchenfunktionäre ausgebildet. In einigen Städten wurden darüber hinaus Bürger- und Realschulen für praktische Berufe errichtet. Das zum Studium berechtigende Examen artium wurde bis 1883 an der Universität abgelegt.

Einer der wenigen bekannteren Mathematiklehrer in Norwegen im 17. Jahrhundert war der aus Kemberg im damaligen Kurfürstentum Sachsen stammende Ambrosius Rhode (Rhodius) (1605-1696). Dieser studierte während des Dreißigjährigen Krieges ab 1627 in Wittenberg Medizin und war ab 1635 in Kopenhagen, wo er auch durch astrologische Veröffentlichungen hervortrat. Ab 1637 war er Stadtphysikus in Christiania (früher und später Oslo) und zugleich Physik- und Mathematiklehrer an der dortigen Kathedralschule, wo später Anfang des 19. Jahrhunderts auch Bernt Michael Holmboe seinen Schüler Abel unterrichten sollte. Über die Mathematikkenntnisse von Rhode wissen wir wenig. Einen größeren Bekanntheitsgrad erlangte er durch Korruptionsbeschuldigungen, die er und seine dänische Frau gegen die damaligen Bürgermeister von Christiania erhoben. Nachdem diese Vorwürfe in einem Prozess nicht bewiesen werden konnten, wurde das Paar 1662 an die nordöstliche Grenze Norwegens nach Vardø 


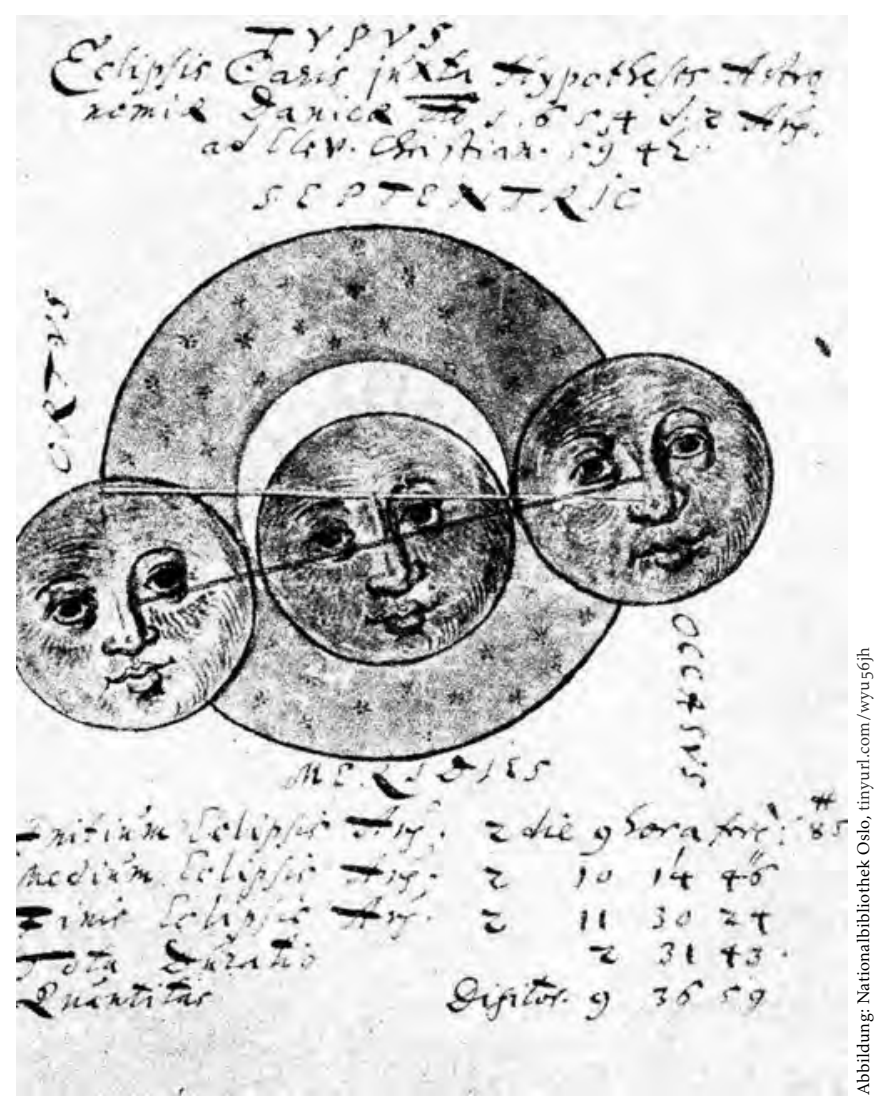

Ausschnitt aus einem astrologischen Manuskript des deutschen Arztes, Naturwissenschaftlers und Mathematiklehrers Ambrosius Rhode (Rhodius) (1605-1696), der lange Jahre an der Kathedralschule in Christiania (Oslo) unterrichtete.

verbannt. Während Rhode 1667 nach Christiania zurückkehren durfte, verstarb seine Frau einige Jahre später in Vardø, er selbst kehrte 1672 in seine Heimatstadt Kemberg zurück.

Erst in der zweiten Hälfte des 18.Jahrhunderts - also schon zu Zeiten Caspar Wessels - sind zwei norwegische Schulmathematiker bekannter geworden, von denen der erste ursprünglich Däne war. Es handelte sich zum einen um Diderich Christian Fester (1732-1811), der 1769 von Kopenhagen nach Trondheim ging und dort Mathematiklehrer an der Kathedralschule wurde. Fester muss sehr vielseitig und originell gewesen sein, wie Brun anmerkt. Letzterer zitiert beispielsweise S. 84 die folgenden Titel von insgesamt 178 Publikationen, allesamt auf Dänisch: Längen, Breiten und Tiefen von Kopenhagens Kanälen und Hafen, Der Vulkan Ätna auf Sizilien, Die Bedeutung der Wissenschaften für die Menschheit, Der Todesgesang eines alten Indianers als die Spanier ihn verbrannten, Grabschrift auf ein eitles und nutzloses Frauenzimmer (Brun 1962, 84). Der norwegische Mathematiker Ralph Tambs Lyche (1890-1991) lobt in einem biographischen Artikel über Fester dessen zwei große Abhandlungen (1781 und 1788) über die Natur des Nordlichtes, die letztere veröffentlicht in den Abhandlungen der Königlichen Akademie der Wissenschaften in Trondheim:
Sein Ergebnis ist, dass das Nordlicht am häufigsten etwa 70 schwedische Meilen, also ca. $100 \mathrm{~km}$ über der Erde auftritt. Das liegt gewiss über den Höhen, die Størmer angibt, aber das Resultat ist für die damalige Zeit nicht schlecht. ${ }^{3}$

Dagegen kommt Tambs Lyche zu dem Ergebnis, dass ein in Trondheim vorhandenes „Vorlesungsmanuskript" Festers, das „eine ziemlich detaillierte Einführung in die Differential- und Integralrechnung" (Tambs Lyche 1935, 3o) enthalte, wahrscheinlich niemals vorgetragen worden sei, da damals in Trondheim weder an der Kathedralschule noch an der Akademie Publikum dafür vorhanden gewesen wäre. Tatsächlich weist Christensen auf einen Rückschlag der Mathematikausbildung infolge der neuen Schulordnung von 1775 hin. Danach wurde an allen Schulen die Ausbildung in Religion und Muttersprache gestärkt. Selbst an den Kathedral- und Lateinschulen sollten dagegen die Mathematik als Pflichtfach entfernt und statt dessen spezielle Rechenlehrer angestellt werden (Christensen 1895, 73). Diese Beschränkungen scheinen sich jedoch nicht voll durchgesetzt zu haben, wie das Reglement für Kathedralschulen in Christiania und Kopenhagen 1801 zeigt. Es heißt dort

Schwerpunkt ist hier die gründliche und beweisorientierte Präsentation der reinen Mathematik, jedoch ohne höhere Analysis. Über die Wissenschaften, die die angewandte Mathematik ausmachen, soll nur ein allgemeiner Überblick gegeben werden, der Rest soll der Universität vorbehalten werden. (Christensen 1895, 57)

Dass damals auch an den höheren Schulen kein Platz für Unterricht in Infinitesimalrechnung war ist selbstverständlich. Diese Beschränkung betraf auch den zweiten Pionier der Schulmathematik im Norwegen des 18 . Jahrhunderts, Frederich Christian Holberg Arentz (1736-1825). Arentz war aus dem norwegischen Bergen gebürtig, seine Mutter war Enkelin des norwegischen und dänischen Nationaldichters Ludvig Holberg, der selbst aus Bergen stammte (Brun 1962, $77 \mathrm{ff})$. Sein Vater, der spätere Bischof von Bergen, beeinflusste Arentz' ursprüngliche Berufswahl und ermutigte ihn zu seinem Theologiestudium in Kopenhagen. Jedoch soll Arentz, dem Mathematiker Brun zufolge, sein schlechtes Gedächtnis davon abgehalten haben, Pfarrer zu werden, weil es verboten war, Manuskripte mit auf die Kanzel zu nehmen. So wurde er schließlich 1762 nach Studien in Göttingen und Leiden Mathematiklehrer an der Kathedralschule in Bergen, deren Rektor er dann seit 1781 gewesen ist.

Auch in der und mit der Mathematik hatte Arentz das Bestreben, überflüssiges belastendes Memorieren zu vermeiden. In diesem Lichte müssen wohl zwei dänisch/ norwegische Veröffentlichungen von Arentz in der Trondheimer Wissenschaftsakademie gesehen werden: „Vorschlag für eine generelle und kürzere Art vernünftige Schlüsse zu ziehen“ (1784) und „Untersuchung, wie man auf die kürzeste Weise gegebene Gleichungen lösen kann, die mehrere oder viele unbekannte Größen enthalten“ (1788). Besonders der letztgenannten Arbeit muss man größere Bedeutung zuerkennen, weil Arentz hier eine Methode benutzt, die der 


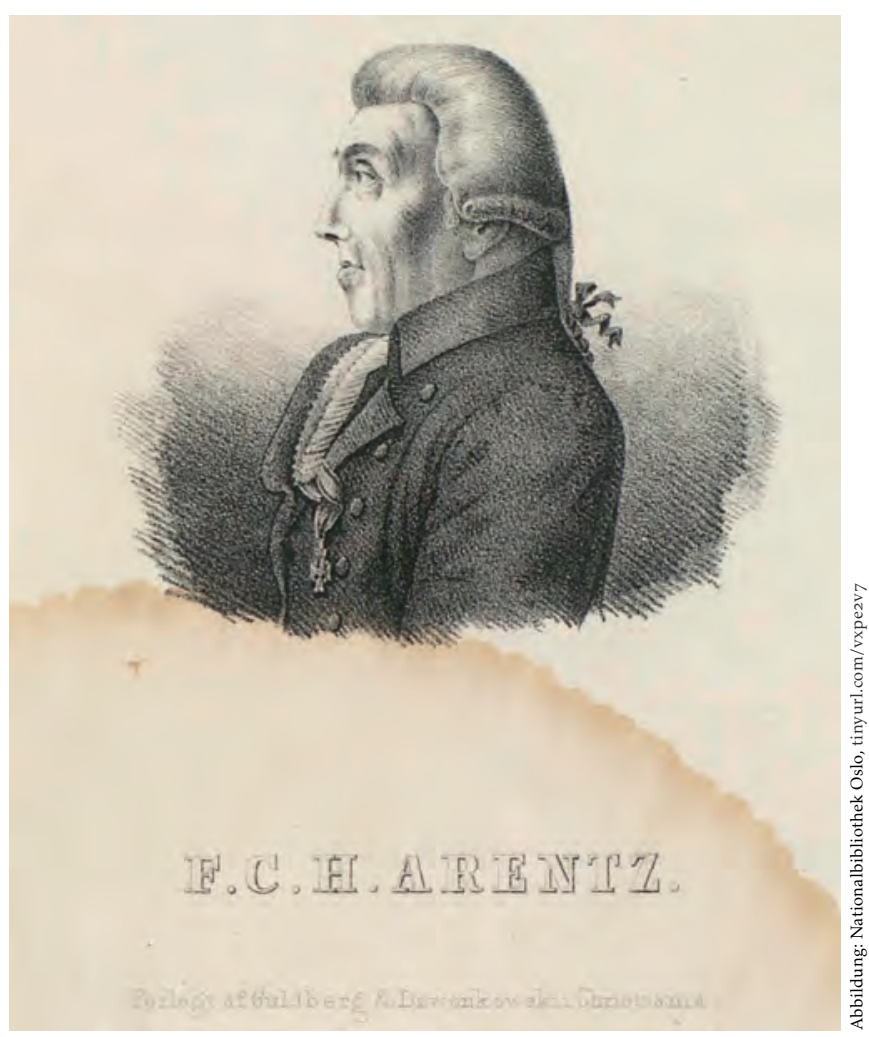

Frederich Christian Holberg Arentz (1736-1825), Mathematiker und Rektor Kathedralschule in Bergen seit 1781 . Unabhängige Entdeckung der Cramerschen Regel für die Auflösung linearer Gleichungssysteme

Verwendung von Determinanten entspricht. Arentz sagt in der Arbeit (zitiert nach Brun 1962, S. 79):

Da ich weder bei Wolff, Clairaut, Euler, Segner oder anderen eine leichtere Methode als die übliche gefunden habe, hielt ich es für nützlich, eine kürzere Art für die Lösung des Problems zu finden.

Es handelte sich hier um die Regel für die Auflösung linearer Gleichungssysteme, die von dem Schweizer Gabriel Cramer bereits $175^{\circ}$ veröffentlicht worden war. Es ist aber gut verbürgt, dass Arentz diese Arbeit nicht kannte.

Zwei weitere interessante Arbeiten von Arentz, die dieser 1779 ebenfalls in seiner Muttersprache in der Trondheimer Wissenschaftsakademie veröffentlichte und in denen er theologische und mathematische Argumente verbindet, hat der Däne Peter Øhrstrøm 1985 untersucht. Arentz wendet sich hier gegen den Begriff der aktualen (wirklichen) Unendlichkeit sowohl in der Mathematik als auch in der Kosmologie, was nicht ausschließe, dass die Existenz der Welt ständig (potentiell) von Gott verlängert werde. Arentz diskutiert in seiner Begründung Grenzwerte und Differentiale und ist in seiner Argumentation ganz offensichtlich von dem ebenfalls in Norwegen geborenen Philosophen und Mathematiklehrer Jens Kraft beeinflusst, der an der Akademie in Sorø wirkte (siehe unten) und sich seinerseits auf Leibniz bezogen hatte. Die zweite der Arentzschen Arbeiten trägt den Titel: „Es ist unmöglich, dass die Welt unendlich sei, weder nach Zeit noch nach Raum. Bewiesen mit mathematischen Gründen." Hier ist die Schlussfolgerung:

All dies betrachtet kann die Welt ohne Ende existieren, genau so, wie eine mathematische Grösse nach Gottes Gefallen ständig ausgedehnt werden kann, ohne doch unendlich groß zu werden (Øhrstrøm 1985, 178)

Wissenschaftsinstitutionen und Mathematik in Dänemark/Norwegen vor Abel

Bevor 1479 die Universität in Kopenhagen gegründet wurde, mussten Studenten aus Norwegen sich in weiter entfernten Universitäten einschreiben. Es gab Norweger unter den Studenten der frühesten europäischen Universitäten in Bologna und Paris im 12. Jahrhundert. Seit dem 15. Jahrhundert war die 1419 gegründete Universität in Rostock ein populärer Studienort, wo es eine eigene norwegische Studentenvereinigung und ein norwegisches Studentenheim gab. Später wurde es dänischen und norwegischen Studenten verboten im Ausland zu studieren, bevor sie nicht grundlegende Fächer in Kopenhagen belegt hatten [Collett 1999, 12], aber nicht alle scheinen sich daran gehalten zu haben (Brun 1962, 48).

Eine Alternative für ein Universitätsstudium in der Mathematik bot die Akademie in Sorø, etwa $80 \mathrm{~km}$ von Kopenhagen entfernt zentral auf der Hauptinsel Seeland (Sjælland). Diese Akademie war 1623 von Christian IV. gegründet und auch mit einem Professorat in Mathematik ausgestattet worden. Unter den Studenten waren vor allem Söhne von dänischen Adligen. Sie wurde 1665 geschlossen und 1747 wiedereröffnet, wesentlich unterstützt durch eine Stiftung von Holberg. Christensen berichtet 1895 in seiner Geschichte der Mathematik in Dänemark und Norwegen im 18. Jahrhundert, dass die Akademie in Sorø stärker forschungsorientiert war als die Universität in Kopenhagen, der maßgeblich das Examenswesen zufiel. Insbesondere der in Norwegen in Fredrikshald (dem heutigen Halden) geborene Philosoph und Naturwissenschaftler Jens Kraft (1720-1765) war in Sorø einflussreich (Brun 1962, 87ff.). Sein dänisch geschriebenes zweibändiges Hauptwerk „Vorlesungen über Mechanik" sammelte viele zeitgenössische mathematische und physikalische Kenntnisse. Darüber hinaus schrieb Kraft eine mehrteilige algebraische Arbeit (17481751) über die Lösung von algebraischen Gleichungen mit Hilfe von unendlichen Potenzreihen. Diese Arbeit benutzte die neuesten Resultate der englischen Analytiker, Brun hat ihr „beachtlichen mathematischen Wert" zuerkannt (Brun 1962, 90) und der dänische Mathematiker und Historiker Niels Nielsen schreibt:

Allein mit dieser Arbeit ragt er als einsamer Kämpe aus der damaligen heimischen mathematischen Mittelmäßigkeit heraus. ${ }^{4}$

Der deutsche Geograf und Theologe Anton Friedrich Büsching (1724-1793), der zeitweise in Kopenhagen lebte, sag- 
te über Kraft 1756 in seinen „Nachrichten von Zustande der Wissenschaften im dänischen Reiche":

Man kennt diesen gelehrten Normann [also Norweger!] als einen starken Philosophen und großen Mathematiker. (Brun 1962, 88)

Im 18. Jahrhundert wurden zwei königliche Wissenschaftsakademien in Dänemark-Norwegen begründet, nämlich Det Kongelige Danske Videnskabernes Selskab i København im Jahre 1742 und Det Kongelige Norske Videnskabers Selskab i Trondheim im Jahre 1760. Die letztere sollte den weit (über $1000 \mathrm{~km}$ ) von der Hauptstadt Kopenhagen entfernten Wissenschaftlern einen Treffpunkt bieten. Eine Akademie im nur halb so weiten Christiania war dagegen nicht so dringend, zumal eine Universität dort damals nicht existierte. Die heutige norwegische nationale Wissenschaftsakademie wurde deshalb erst 1857 gegründet. Wir haben weiter oben von Festers und Arentz' Publikationen in den Abhandlungen der Trondheimer Akademie berichtet, die auch heute weiter besteht.

1750 wurde in Christiania die „Freie Mathematische Schule“ (Den frie matematiske skole) gegründet, die bis 1798 bestand und Vorgängerin der Königlichen Kriegsschule wurde, der ersten höheren akademischen Ausbildungsstätte für norwegische Offiziere. Hier wurde besonderes Gewicht auf Mathematik, Festungsbau, Artillerie, sowie Architektur und technische Zeichnung gelegt. Nach einhelligem Urteil der Historiker (Christensen, Brun) ist das mathematische Niveau dort aber zumindest im 18. Jahrhundert recht niedrig gewesen. Kreative Mathematiker unter den Lehrkräften sind nicht bekannt geworden.

Im Jahre 1757 wurde die Bergwerksakademie in Kongsberg, $80 \mathrm{~km}$ südwestlich von Christiania-Oslo gegründet, im Zusammenhang mit der Erzgewinnung dort, die auch mathematische Kompetenz erforderte. Als um 1810 noch unter dänischer Vorherrschaft Pläne für die Gründung einer norwegischen Universität gemacht wurden, war Kongsberg ein plausibler Ort. Viggo Brun $(1962,69 / 70)$ beschreibt anschaulich und phantasievoll, wie man in Kongsberg 1811 die Beförderung zur Universitätsstadt feierte. Einige Monate später wurde dann aber die Königliche Fredriks Universität, wie sie bis in die $1930 e r$ Jahre heißen sollte, dennoch in Christiania gegründet, und die Kongsberger Bergakademie wurde schließlich in diese integriert.

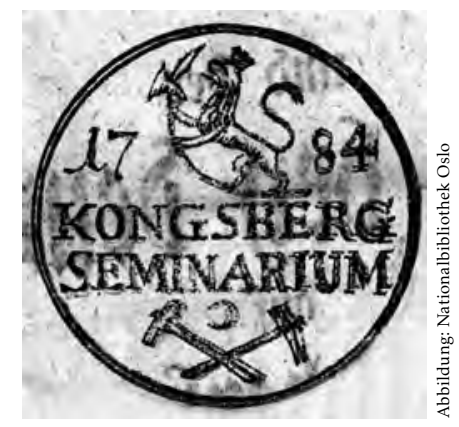

Siegel der $175^{1}$ gegründeten Bergakademie Kongsberg (8o km von Christiania), wo Mathematik im Bergwesen angewandt wurde.
Caspar Wessel (1799) und das Ende der dänischen Zeit für die norwegische Mathematik

Der ohne Vergleich originellste und interessanteste norwegische Mathematiker vor Abel war Caspar Wessel. Er wuchs in Vestby bei Christiania in einer Pfarrersfamilie mit 13 Kindern auf. Sein Bruder Herman Wessel (1742-85) wurde als Dichter berühmt und war eine zentrale literarische Gestalt im Dänemark-Norwegen der Aufklärungszeit. Caspar studierte in Kopenhagen, arbeitete als Landmesser in Dänemark und zeitweise im Herzogtum Oldenburg bei Bremen. Gauß hat übrigens später 1824 bei seinen Triangulationen auch an Wessels Messungen in Oldenburg angeknüpft (Branner/Johansen 1999, 42). Wessel entwickelte im Zusammenhang mit seinen Landmessungen einen Kalkül für das Rechnen mit ebenen und sphärischen Polygonen, die einer ersten geometrischen Darstellung komplexer Zahlen entsprach. Eine eindrucksvolle Beschreibung der Person Wessels - von dem anscheinend kein Porträt existiert - ist in deutscher Sprache überliefert. Dies in einem Brief des Kopenhagener Professors für Astronomie Thomas Bugge (1740-1815) an den aus Bayern stammenden Stiftamtmann in Oldenburg, den Ökonomen und Botaniker Georg Christian Oeder (1728-1791). Dieser stand zeitweise in dänischen Diensten und hatte selbst erhebliche wissenschaftliche Verdienste als Verfasser der Flora Danica seit den $1750 e r$ Jahren. Er war 1773 nach Oldenburg gekommen, als dieses noch dänisch war. Bugge schreibt am 25. Dezember 1781 an Oeder über Wessel:

Er ist ein Mann von mittlerer Länge, mager und schwarzer (Haar-)Farbe. Sein Gesicht trägt Spuren scharfen Denkens und zeigt die ernsthaften Züge, welche bei einem Mann, der viel observiert und rechnet, sich einfinden müssen. Er besitzt viel theoretische Kenntniss besonders in der Algebra, Trigonometrie und mathematischen Geometrie, in welcher letzten er neue und schöne Auflösungen der schwersten, die geographische Landmessung betreffenden Aufgaben, gegeben hat. Er spricht wenig, aber sehr wohl, er ist in seinem Wesen zurückhaltend, aber dabei weder arg noch Sonderling. Er ist bescheiden und denkt immer von seinen eigenen Einsichten und Arbeiten gering und ist im Stande, indem er wirkliche Meisterstücke des Fleisses, der Kunst und der Einsicht vorlegt, Ihnen zu sagen, dass es nicht viel auf sich habe. (Branner/Johansen 1999, 41)

Wessels einzige mathematische Abhandlung wurde separat (1798) und danach in den Schriften der Königlichen Dänischen Akademie der Wissenschaften in Kopenhagen veröffentlicht [1799], blieb aber in mathematischen Kreisen für etwa einhundert Jahre weitgehend unbekannt. Dies kann zweifellos teilweise durch die Sprachbarriere und dadurch erklärt werden, dass Wessel nicht in einem zur damaligen Zeit mathematisch tonangebenden Land wirkte. Es war anscheinend Christensens Doktorarbeit von 1894 über die Entwicklung der Mathematik in Dänemark und Norwegen, veröffentlicht als [Christensen 1895], die Wessels Arbeit in 


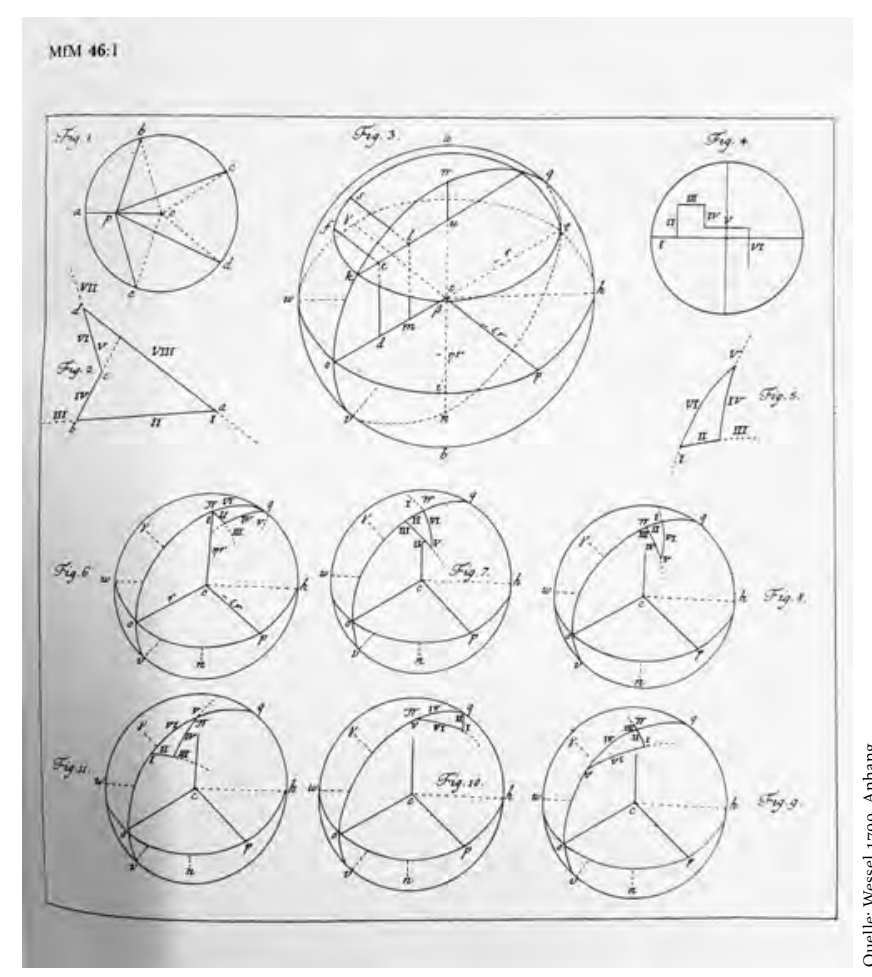

Beispiele für die Ausdehnung von Wessels Kalkül für Polygone auf der Kugeloberfläche

Erinnerung brachte. Der große Norweger Sophus Lie druckte die Arbeit, die bald auch von der dänischen Akademie in französischer Übersetzung publiziert wurde [Wessel 1897], noch 1895 in dem von ihm mitherausgegebenen Archiv for Mathematik og Naturvidenskab neu ab (Brun 1962, 106). Eine reich kommentierte englische Übersetzung erschien schließlich ein weiteres Jahrhundert später als [Wessel 1999].

Wessels Ziel war es, Richtung und Länge unbekannter Seiten von Polygonen, insbesondere von Dreiecken, zunächst solcher in der Ebene, zu bestimmen. Er entwickelte dafür einen Kalkül der Addition und Multiplikation von koplanaren Liniensegmenten. Wessel führt die Repräsentation $\cos v+\varepsilon \sin v$ für beliebige Liniensegmente in der Ebene ein, wo $v$ die Richtung, d.h. den Winkel mit einem vorgegebenen Einheitsvektor bedeutet und $\varepsilon$ dem imaginären Element $\sqrt{-1}$ entspricht. Aber Wessel geht noch weiter und entwickelt den Kalkül auch für Polygone auf der Kugeloberfläche. Die Rechenregeln entsprechen dabei der komplexen Arithmetik in jeder der drei Projektionsebenen.

Der im Alter zunehmend empfindliche und ehrbegierige (besonders gegenüber seinem früheren Freund Felix Klein) sowie sich norwegisch patriotisch äußernde Sophus Lie schien übrigens bei seinen Bemühungen um Wessel zu unterstellen, dass die Dänen die norwegische Herkunft Wessels verschweigen wollten. Lie schreibt im Dezember 1896 in einem Brief an Ludvig Sylow:

Ich habe mich sehr darüber geärgert, dass die Dänen sich C. Wessel zu eigen machen wollten. Das erscheint mir wahrlich kläglich. Eigentlich war es

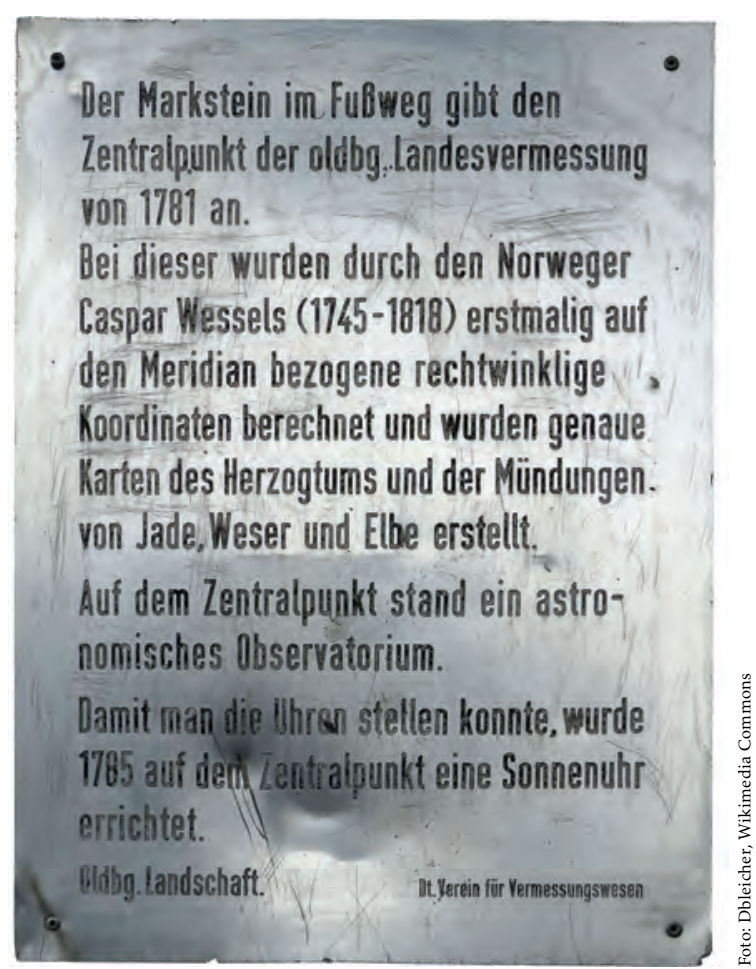

Gedenkplakette für die oldenburgische Landesvermessung im Schlossgarten in Oldenburg. Caspar Wessel(s) Name ist hier in den Genitiv verfälscht.

ja ein großes Unglück für Wessel und die norwegische Wissenschaft, dass dieser begabte Mathematiker sich in einer Umgebung befand, der es an jeglichem Verständnis für ihn mangelte. Und die Art und Weise, wie man Wessels Abhandlung endlich entdeckte, spricht nicht gerade für die dänische Mathematik. Du kennst doch Christensens und Juels Anteil an der Geschichte. Die Geschichte ist die alte: Die Dänen litten zeitlebens unter den Deutschen und selbst erlauben sie sich dieselben Übergriffe, für die sie die Deutschen anklagen, bei den Norwegern. (Stubhaug 2003, S. 522)

Dies klingt allerdings ziemlich unplausibel und überpatriotisch, gerade weil Christensen (1895) erwähnte, dass Wessel in Norwegen geboren worden war.

Wir schließen unsere kurze Beschreibung von Wessel und damit auch unseren Aufsatz über die norwegische Mathematik unter den Dänen mit einem Ausschnitt aus einem Schreiben, das die dänische Akademie der Wissenschaften, vertreten durch die Mathematiker J. Thomsen und H. Zeuthen, 1897 zusammen mit der französischen Übersetzung der Wesselschen Arbeit an die relativ junge norwegische Akademie der Wissenschaften in Kristiania (später Oslo) schickte. Die komplizierte diplomatische Logik darin möge zugleich als ein Resümee der komplizierten Beziehungen zwischen dänischer und norwegischer Mathematik in dem von uns betrachteten Zeitabschnitt dienen. Es kann außerdem als eine Überleitung zu der nächsten Periode norwegischer Mathematik verstanden werden, die wesentlich 
von Abel geprägt wurde, auf den das Schreiben am Ende anspielt:

Eine Einteilung der großen Norweger und Dänen nach ihrem Geburtsort würde für die Zeit, als die beiden Reiche vereint waren, einen Bruch der Gemeinschaft bedeuten, die fruchtbar und nützlich für beide Länder gewesen ist. Vor allem wäre es ein Zeichen der Undankbarkeit gegenüber den Norwegern, denen unser eigenes Geistesleben so viel schuldet. Es sollte umso weniger vergessen werden, dass Norwegen Wessels Geburtsland war, weil dieses Land, wo Wessel bald von einem der allergrößten Mathematiker des Jahrhunderts gefolgt wurde, weiterhin eine bedeutende Stellung in derjenigen Wissenschaft einnimmt, die diese beiden Männer prägte. (Zitiert nach Brun 1962, 10)

\section{Anmerkungen}

1. Hans Gram (1685-1748), dänischer Historiker, 1734 über die Norweger, möglicherweise auch gemeint als indirekte Kritik an dem gleichaltrigen, viel bekannteren Schriftsteller und Philosophen Ludvig Holberg (1684-1754) aus dem norwegischen Bergen. Dies ist unsere Übersetzung des originalen Textes in (Brun 1962, 59).

2. Seit der Loslösung Norwegens von Dänemark 1814 hat sich die norwegische Sprache in der Schreibweise, vor allem aber in der Aussprache wieder etwas vom Dänischen entfernt.

3. Tambs Lyche $(1935,32)$. Tambs Lyche spielt hier auf die bekannte vielseitige Nordlichtanalyse durch den Mathematiker Carl Størmer (1874-1957) an.

4. (Nielsen 1912, 301). Nielsen geht auch in einem gesonderten $\S 13$ „Nebenbeschäftigungen der Professoren. Nepotismus" (251-254) auf die „Vererbung" von Professuren zum Beispiel in den Familien Bartholin und Horrebow ein.

\section{Literatur}

Branner, B. und N.V. Johansen (1999): Caspar Wessel (1745-1818). Surveyor and Mathematician, in: Wessel (1999), 9-61.

Brun, V. (1962): Regnekunsten i det gamle Norge. Oslo-Bergen.

Christensen, S.A. (1895): Matematikens udvikling i Danmark og Norge $i$ det XVIII. aarhundrede. Odense 1895 .

Collett, P. (1999): Historien om Universitetet $i$ Oslo. Oslo.

Nielsen, N. (1912): Matematiken i Danmark 1528-180o. Kopenhagen 1912.

Nossum, R. und R. Siegmund-Schultze (2011): Mathematik in Norwegen II: Von den Anfängen im Mittelalter bis zum 14. Jahrhundert, Mitteilungen der DMV 19, 49-55

Siegmund-Schultze, R. (2004): Mathematik in Norwegen I: Ein Land mit „sehr wenig Poeten und allzu vielen Mathematikern"?, Mitteilungen der $D M V$ 12, 36-40.

Stubhaug, A. (2003): Es war die Kühnheit meiner Gedanken. Der Mathematiker Sophus Lie. Berlin: Springer.

Tambs Lyche, R. (1935): Matematikkens stilling i Norge omkring ${ }^{17} 80-$ arene, belyst ved D. C. Festers virksomhet i Trondheim, Det kgl. Norske vid.selsk. Forhandlinger VII, $26^{*}-33^{*}$.

Wessel, C. (1799): Om Directionens Analytiske Betegning. Det Kgl. Danske Videnskabers Selskabs Skrifter. 5, 469-518, und Archiv for Math. og Naturvidenskab 18 (1896), 5-69. (Mit einer Vorrede von Sophus Lie, pp. 3/4).

Wessel, C. (1897): Essai sur la représentation analytique de la direction. (Ed. H.Valentiner und T. N. Thiele), Dänische Akademie der Wissenschaften), Kopenhagen.

Wessel, C. (1999): On the Analytical Representation of Direction. Copenhagen Reitzel (Edited by Bodil Branner and Jesper Lützen).

Øhrstrøm, P.: F. C. H. Arentz' matematiske argumenter mod verdens uendelighed i tid og rum, Normat 33 (1985), 176-185.

Mit diesem Beitrag setzen die Autoren eine Reihe von kurzen Mitteilungen (Siegmund-Schultze 2004, Nossum und Siegmund-Schultze 2011) über die Geschichte der norwegischen Mathematik in den Mitteilungen der DMV fort.

Prof. Dr. Rolf Tomas Nossum, University of Agder, Faculty of Engineering and Science, Department of Mathematical Sciences, Gimlemoen 25a, Postboks 422, 4604 Kristiansand S, Norwegen rolf.nossum@uia.no

Prof. Dr. Reinhard Siegmund-Schultze, University of Agder, Faculty of Engineering and Science, Department of Mathematical Sciences, Gimlemoen 25a, Postboks 422, 4604 Kristiansand S, Norwegen reinhard.siegmund-schultze@uia.no

Rolf Tomas Nossum ist 1954 in Barum, Norwegen, geboren. Er hat in Oslo studiert und dort 1984 zur Komplexität der Verifikation von Programmen promoviert. 2004 erhielt er eine Professur für Informatik in Kristiansand, wo er sich auch mit der Geschichte der Mathematik beschäftigt.

Reinhard Siegmund-Schultze ist 1953 in Halle geboren, wo er Mathematik studiert und 1979 zur Geschichte der Funktionalanalysis promoviert hat. Im Jahre 2000 erhielt er eine Stelle als Mathematikhistoriker in Kristiansand. Er schreibt gegenwärtig an einer Biografie des angewandten Mathematikers und Ingenieurs Richard von Mises (1883-1953). 